\title{
Narrative niche construction: memory ecologies and distributed narrative identities
}

\author{
Richard Heersmink ${ }^{1}$
}

Received: 24 February 2020 / Accepted: 18 September 2020

(c) Springer Nature B.V. 2020

\begin{abstract}
Memories of our personal past are the building blocks of our narrative identity. So, when we depend on objects and other people to remember and construct our personal past, our narrative identity is distributed across our embodied brains and an ecology of environmental resources. This paper uses a cognitive niche construction approach to conceptualise how we engineer our memory ecology and construct our distributed narrative identities. It does so by identifying three types of niche construction processes that govern how we interact with our memory ecology, namely creating, editing, and using resources in our memory ecology. It also conceptualises how identity-relevant information in objects and (family) stories is transmitted vertically, i.e., across generations of people. Identifying these processes allows us to better understand the cultural information trajectories that constitute our memory ecologies. In short, what I'll argue is that our memory ecology scaffolds our narrative identity and that engineering our memory ecology is a form of narrative niche construction.
\end{abstract}

Keywords Narrative identity · Distributed self · Extended self · Cognitive niche construction · Cognitive ecology · Distributed cognition · Transactive memory • Extended mind

\section{Introduction}

We often remember our personal past through interacting with objects (e.g., photos, journals, mementos, lifelogs) and reminiscing with other people (e.g., spouses, friends, colleagues). Such objects and people involved in autobiographical remembering constitute our memory ecology. Human autobiographical memory is open to incorporate and rely on resources in our memory ecology, in that way our memory systems are distributed across embodied brains and environmental resources

Richard Heersmink

richard.heersmink@gmail.com

1 Politics, Media and Philosophy, La Trobe University, Melbourne, Australia 
(Wegner 1986; Hutchins 1995a; Clark and Chalmers 1998; Michaelian and Sutton 2013). This has important consequences for our narrative identity because autobiographical memories are the building blocks of our narrative. An influential view on personal identity is the narrative self-constitution developed by Schechtman (1996, 2005). This view suggests that our identity and self are constituted by our autobiographical narrative. What makes you the particular person you are and what distinguishes you from other persons is your unique autobiographical narrative. So, in an important sense, we are our narrative, which can be defined as a subjective, affective, and personal story containing a mostly accurate chronological depiction of a series of connected events and experiences that constitute our identity. The upshot of integrating the distributed memory thesis and the narrative self-constitution view is this: If the memories realising our narrative identity are distributed across our brains, bodies, and memory ecology, then our narrative identity is distributed, too (Heersmink 2017, 2018, 2020).

We don't yet fully understand the various processes constituting how we interact with and construct our memory ecology. Most research, including my own, has looked at the informational properties of objects (Donald 1991; Hutchins 1999; Heersmink 2013, 2016) but has not paid much attention to interactional processes (but see, e.g., Bietti and Sutton 2015; Steffensen 2017; Fasoli 2018). This paper therefore aims to conceptualise how we interact with and engineer our memory ecology by using a niche construction approach. Niche construction theory is an approach in evolutionary biology, focussing on how organisms change and utilise the environment as to improve their fitness landscapes (Laland et al. 2000; OdlingSmee et al. 2003). Changing the environment allows niche constructing organisms to influence selection pressures on certain biological, behavioral, and cognitive traits. This theory departs from traditional evolutionary theory, in that it suggests there are two main inheritance mechanisms, one genetic and the other ecological. Ecological inheritance includes the transfer of knowledge, skills, artifacts, and institutions (Jablonka and Lamb 2005). Cognitive niche construction looks specifically at how humans create cognition-aiding tools (e.g., an abacus or computer) and representational systems (e.g., language or number systems) to realise their cognitive goals (Sterelny 2003, 2010; Clark 2006). It also looks at how these tools and representational systems are transmitted across generations.

In this paper, I will analyse how we interact with and construct our memory ecology by using the lens of cognitive niche construction theory to look at empirical research in cognitive science and human-technology interaction. I shall conceptualise how identity-relevant information in our memory ecology is actively created, edited, and used. I also conceptualise how identity-relevant information in objects and (family) stories is transmitted vertically, i.e., across different generations of people, which can be seen as a form of downstream narrative niche construction. Most theorizing on cognitive niche construction is about how humans solve practical problems, ignoring autobiographical remembering (Sterelny 2003, 2004, 2010, 2014; Clark 2006; Stotz 2010; Kendall 2011; Sinha 2015; Bertolotti and Magnani 2017; Heras-Escribano 2020). One goal of this paper is to draw attention to and further develop the relation between autobiographical remembering and niche construction theory. 
This paper has the following structure. In the next section, I start by introducing the notion of a memory ecology and argue that autobiographical memory systems are sometimes distributed across embodied agents and their memory ecology. In "Distributed narrative identities" section, I draw out the implications of this claim for our narrative identity, suggesting that narrative identity is a relational and distributed structure. In "Niche construction" section, I outline the niche construction approach in evolutionary biology, focusing on cognitive niche construction. Having thus set the stage, I'll apply a cognitive niche construction approach to analyse how we interact with and engineer our memory ecologies and maintain our distributed narrative identities in "Narrative niche construction" section.

\section{Memory ecologies}

\section{Evocative objects}

Turkle (2007) introduces the notion of an "evocative object", which she characterises as objects that generate a feeling or thought (or both) in their user. Turkle's (2007) edited book contains various examples of singular objects such as a car, suitcase, cello, radio, painting, analogue camera, raincoat, glucometer, and many more personal objects. These objects are evocative because they make their users feel and think a certain way, typically associated with their personal past. Their evocative powers sometimes aren't the primary or intended function of the object but more an accidental by-product of using these objects in daily life. For example, a car is primarily designed and used for driving but may also remind its user of a previous roadtrip, a suitcase is primarily designed and used for carrying materials but may also remind its user of a previous citytrip, etc.

I want to stretch Turkle's concept of evocative objects here to go beyond singular, material, tangible structures. Instead, I want the concept to be more liberal, including any product of human agency with evocative powers such as smells, music, moving images, stories, buildings, and larger built structures. Examples of objects evoking personal memories range from perfumes, songs, movies, souvenirs, furniture, works of art, jewellery, books, photo albums, collections of objects, trophies, inherited objects, and other types of mementos. Larger built and public structures such as monuments, churches, trains, and bridges may also evoke memories. Even our embodied presence in places or spaces like restaurants, public squares, streets, parks, and airports can evoke personal memories.

In proposing this notion, Turkle draws attention to the emotional and autobiographical dimensions of artifacts, which haven't received much attention in the philosophical and scientific literature on artifacts. For example, the central works in the philosophy and metaphysics of artifacts (e.g., Dipert 1993; Houkes and Vermaas 2010; Kroes 2012), including major handbooks (Margolis and Laurence 2007; Meijers 2009) and the recently updated entry on "Artifact" in the Stanford Encyclopedia of Philosophy (Preston 2018) don't discuss emotional and autobiographical characteristics of artifacts. Likewise, central works in situated, extended, and distributed cognition theory (Norman 1993; Hutchins 1995a; Clark 2003, 2008), including the 
major handbooks (e.g., Robbins and Aydede 2008; Menary 2010b; Newen et al. 2018) ignore such dimensions of artifacts. So, emotional and autobiographical characteristics of material culture are a neglected topic in philosophy and cognitive science. One goal of this paper is to draw attention to and further conceptualise emotional and autobiographical dimensions of material culture.

We feel connected to evocative objects because they remind us of our past experiences and activities, occasionally also inducing emotional states (Piredda 2020). Emotional states are sometimes part of the content of a memory and so when we retrieve an emotionally-laden memory of a past event, it can make us feel a certain way (Pascuzzi and Smorti 2017). In cognitive psychology, memory processing is divided in three stages: encoding, storing, and retrieving. Experiences are first encoded, then stored in connectionist networks, and later retrieved. Interacting with evocative objects primarily activate cognitive processes which construct and retrieve the contents of stored memories into consciousness. This is related to the more familiar notion of an epistemic action (Kirsh and Maglio 1994). Epistemic actions change the input to an agent's cognitive system and make cognitive processes easier, faster, or more reliable. Using cognitive artifacts to perform a cognitive task (e.g. deploying a map to navigate) are epistemic actions. Intentionally interacting with an evocative object to evoke and construct an autobiographical memory is a type of epistemic action, as such actions make it easier to retrieve memories from our biological memory system. Because the information stored in connectionist networks in the brain fades over time, we need the informational stability provided by the environment to reliably remember our personal past (Sutton 2009). When using objects to remember our past, we don't retrieve and construct a complete internal memory. Rather, information in the brain and in the object is integrated as to construct a personal memory, suggesting that both the vehicles and processes of remembering our personal past are distributed, in that way constituting a new systemic whole (Heersmink 2018). I thus conceptualise human memory systems as extended or distributed across embodied brain and environmental resources. ${ }^{1}$ Extending or distributing our autobiographical memories allows us to remember our personal past in a way that is quite different from remembering our past without the aid of objects, allowing us to remember our personal past in a more reliable and detailed manner.

Recently, new evocative objects are emerging referred to as lifelogging or selftracking systems, which are particularly powerful autobiographical memory technologies. Lifelogging is the process of capturing one's past activities and storing these in a lifelog. This is done with a variety of technologies, including SenseCams, which are small wearable cameras with a wide-angle lens worn around one's neck. These small cameras take a picture every time its internal sensors detect a change in environmental conditions such as GPS location and light intensity, so every time you're in a different situation a photo is taken. At the end of the day, you'll have hundreds of photos representing a visual narrative of one's daily activities. Many

\footnotetext{
1 The extended mind thesis received various criticisms, e.g., by Rupert (2004, 2010) and Adams and Aizawa (2008). This paper is not the place to defend the extended mind thesis, but for responses to these criticisms, see Clark (2008), Menary (2010a) and Rowlands (2010).
} 
lifelogging technologies are embedded in smartphones and other mobile computing devices (e.g., Fitbits and smartwatches), allowing a user to track one's GPS location, sleep patterns, heart rate, dietary intake, purchases, and many other variables. These technologies also provide functionalities for archiving emails, text messages, calendar entries, visited webpages, photos, and videos.

Computer scientist and prominent lifelogger Gordon Bell has created a very extensive lifelog with annotated photos, videos, audio recordings, webpages, GPSbased locations, letters, memos, receipts, business cards, meeting agendas, symposium programs, diplomas, employee evaluations, newspaper clippings, and much more. He and his team also developed software allowing him to easily and quickly search his lifelog (Bell and Gemmell 2009). Bell and Gemmell claim that lifelogs "will become vital to our episodic memory. As you live your life, your personal devices will capture whatever you decide to record. Biomemories fade, vanish, merge, and mutate with time, but your digital memories are unchanging" (2009, p. 57). Precisely because lifelogs are more reliable and accurate than biological memory, they can complement the perceived shortcomings of biological memory such as lack of detail, limited storage capacity, and its unreliability, which is for most users the main reason for using lifelogging technology. Lifelogging and self-tracking are becoming increasingly popular, not just with millennials but across subcultures and demographic groups (Lupton 2016). Thus there seems to be a trend where humans rely more on technology to aid them in remembering their personal past.

\section{Transactive memory systems}

Other people, too, help us remember our personal past. Cognitive psychologist Wegner (1986) develops a theory on how the memory systems of people in close relationships like marriage, kinship, or friendship are intertwined. In such transactive memory systems, there is a cognitive interdependence between group members, in which case both agents rely on each other's memory systems. A transactive memory system can be characterised as a cognitive system comprising people in close relationships in dyads or larger groups who engage collaboratively in encoding, storing, and retrieving information. Consider the following example of transactive retrieval from the empirical work of Harris et al. (2014) in which a long-married couple tries to remember the name of the show they saw on their honeymoon more than 40 years ago.

Wife: And we went to two shows, can you remember what they were called?

Husband: We did. One was a musical, or were they both? I don't... no... one...

Wife: John Hanson was in it.

Husband: Desert Song.

Wife: Desert Song, that's it, I couldn't remember what it was called, but yes, I knew John Hanson was in it.

Husband: Yes.

In this example, two people give each other personalised cues as to trigger and retrieve memories in their transactive memory partner, in that way generating an 
emergent memory system that knows more than the individual members. Typically, the longer people know each other, the deeper their memory systems are integrated, and the more efficient the integrated system works. This is so because they have more shared experiences and a deeper and broader understanding of the epistemic expertise of their transactive memory partner.

How often we rely on information in other people's memory systems can be seen in terms of a spectrum, varying from one-offs (e.g., asking directions to a stranger) to more or less permanent relationships (e.g., with your spouse). Transactive memory systems are those more or less permanent systems that are also deeply integrated, interdependent, and typically well-functioning, in that the emerging memory system is more than the sum of its parts as the Desert Song example shows. In transactive memory systems, encoding processes can be done jointly, for example when a couple negotiates who has to remember a joint appointment. Retrieval processes are integrated and interdependent through interactive cuing. Sometimes retrieved experiences are shared, for example when reminiscing about a past museum visit, citytrip, or honeymoon.

\section{Cognitive ecology}

The embodied brain is a powerful cognitive system, but there are limits to its capacity to retain detailed information over a long period of time. Consequently, for pragmatic reasons, we often rely on information stored in artifacts and other people to complete our memory tasks. Human thinking and remembering thus takes place in a cognitive ecology (Hutchins 2010), which has been characterised as "the multidimensional contexts in which we remember, feel, think, sense, communicate, imagine, and act, often collaboratively, on the fly, and in rich ongoing interaction with our environments" (Tribble and Sutton 2011, p. 94). An underexplored part of our cognitive ecologies are environmental resources involved in autobiographical remembering, which is the focus in this paper. I refer to the total field of evocative objects and transactive memory partners as a memory ecology. Any environmental resource (artifactual and social) involved in autobiographical remembering is part of our memory ecology and an explanans of autobiographical memory.

Distributed cognition theory suggests that human cognitive systems are distributed across embodied agents and environmental structures such as artifacts and people (Hutchins 1995a; Michaelian and Sutton 2013). On this view, cognitive systems aren't merely realised by structures in the brain but emerge from our interactions with environmental resources. The distributed cognition framework "is explicitly cognitive in that it is concerned with how information is represented and how representations are transformed and propagated in the performance of tasks" (Hutchins 1995b, p. 265). This view has both methodological and ontological implications. The methodological upshot of this view is that to better understand how representations are transformed and propagated during tasks, the unit of analysis ought to be enlarged such that environmental resources are part of the explanans of cognitive systems. Better understanding what I will call "information trajectories" is essential to better understanding the workings of distributed cognitive systems, including 
those related to autobiographical memory. The ontological upshot of this view is that cognitive systems are constitutively composed of biological and environmental (i.e., technological and social) components (Clark and Chalmers 1998).

Hutchins, still working in a cognitivist framework, perhaps overemphasizes the importance of the propagation of representations. In case of distributed autobiographical remembering, for example, non-representational evocative objects can contribute to constructing a personal memory. For example, in their ethnographic work on evocative objects, Petrelli et al. (2008) observed and interviewed various participants about their evocative objects. Reflecting on the autobiographical meaning of her coffee mug, one participant says: "I feel very emotionally attached to it for some reason. I bought it in London, when I was working in London. I think it is the memory of working in publishing, living in London and going through a sort of fulfilling patch in my career" (2008, p. 56). This coffee mug is not an external representation in that it doesn't contain representational information such as photos, language, or symbols. It nonetheless reminds its owner of certain life periods. Many evocative objects are, likewise, non-representational objects obtaining their autobiographical function through accidentally associating the object with a memory (Kirk and Sellen 2010). Finally, I agree with Hutchins that "the study of cognitive ecosystems will become an increasingly important part of cognitive science" (2010, p. 705). In this paper, I aim to develop the notion further by homing in on one part of our cognitive ecosystem, namely the part that is involved in autobiographical remembering.

\section{Distributed narrative identities}

\section{Narrative selves}

Schechtman $(1996,2005)$ argues that our identity is constituted by our self-narrative. On this view, what makes you the particular person you are and what distinguishes you from other persons is your unique autobiographical narrative. So when characterising our identity, we need to focus on our autobiographical memories and how they build up a unique narrative of our past experiences and activities. According to Schechtman (1996), in order for a narrative to be identity-constituting, it needs to satisfy three criteria or constraints: the narrative needs to be mostly linear, a person needs to be able to articulate or think through (parts of) the narrative, and it has to be largely consistent with reality. Human lives unfold chronologically and so the narrative must reflect this in order to be truth-preserving. If we remember our personal past non-chronologically, it doesn't reflect actual events and the causal relations between them. On the articulation constraint, a person has to be able to articulate or think through some version of one's narrative. When pressed, most of us would be able to think through, articulate, or write down a reasonably elaborate and detailed autobiography. We know where we lived, studied, worked, travelled, who our family, friends and colleagues are, who we've dated, etc. On the reality constraint, which is related to the linearity constraint, the events constituting the narrative must be mostly truthful. So, delusions and confabulations can't be 
identity-constituting. However, because autobiographical memory is prone to error (Schacter 2001) and can be manipulated (Loftus 2003), narratives rarely are perfectly accurate depictions of our past.

An essential property of a self-narrative is emplotment. In Paul Ricoeur words "emplotment is the operation that draws a configuration out of a simple succession" (Ricoeur 1984, p. 65). During the process of emplotment, we create meaningful causal connections between the building blocks of the narrative which are the autobiographical memories. This happens both on a conscious and subconscious level. We sometimes actively create links between memories by thinking through and analysing past events. But emplotment also happens below the threshold of consciousness, for example during sleep, when our brain processes experiential information, integrating it into our narrative and web of beliefs. Without emplotment, there would only be a mere succession of events without an understanding of how they are causally related. Emplotment is thus the glue that keeps the building blocks of the narrative together. I characterise a self-narrative as a subjective, affective, and personal story containing a mostly accurate chronological depiction of a series of connected events and experiences that constitute our identity.

Strawson (2004) has written an influential criticism on the notion of narrative selfhood. He distinguishes between a descriptive and ethical version of the narrative self. The descriptive version claims that, as an empirical fact, people have a narrative sense of their past and lives, whereas the ethical version claims that conceiving of one's life narratively is desirable, contributing to a well-lived life and full personhood. Strawson disagrees with both claims. He attacks narrative theorists by saying that their theories are based merely on introspection. When narrative theorists introspect, they find a narrative that structures their lives and experiences. They then generalise this observation to all persons. When Strawson himself introspects, he finds no narrative, providing a counter example to the universalist claims of narrative theorists. My view is that narratives being implicit structures doesn't undermine their role in constituting identity. I don't constantly have to think through my past and narrative for it to be constitutive of my identity (Jongepier 2016). Furthermore, there is empirical evidence supporting the narrative self view (e.g., Habermas et al. 2009). There are also more conceptual defences against Strawson's criticism (e.g., Rudd 2009; Mackenzie and Poltera 2010; Schechtman 2014). ${ }^{2}$

\section{Relational identities}

How do memory ecologies and autobiographical narratives interact? Memory ecologies help us to remember our personal past, which is important because the way we remember our personal past is constitutive of our narrative identities. The

\footnotetext{
2 Additionally, I don't deny that persons also have a minimal self or a subject of experience (Gallagher 2000). This is the entity that has experiences and initiates action and so it has subjecthood and agenthood. My view is that some of the experiences of the minimal self ultimately become memories that are integrated into the narrative constituting our identity. But for more discussion on the relation between the minimal and narrative self, see (Menary 2008; Zahavi 2010; Krueger 2011; Heersmink 2020).
} 
autobiographical memories realising our narrative identity are thus distributed across our embodied brains and memory ecologies. Without interacting with our memory ecology, we wouldn't be able to remember our past in the same way. Our memory ecology thus provides stability for our narrative identity. The argument I'm proposing has the following structure:

(P1). Human selves have a narrative structure realised by autobiographical memories.

(P2). Some of our autobiographical memories are distributed across embodied brains interacting with their memory ecology.

(P3). If human selves have a narrative structure realised by autobiographical memories and if some of our autobiographical memories are distributed, then human selves are also distributed.

(C). Therefore, human narrative selves are sometimes distributed structures.

In Turkle's (2007) edited volume on evocative objects, design theorist William Mitchell suggests a similar view when he writes that "objects, narratives, memories and space are woven into a complex and expanding web, each fragment of which gives meaning to all the others" (Mitchell 2007, p. 150). I think Mitchell is right in saying that objects, narratives, memories, and space are integrated into a larger entity. On my view, this larger entity is our distributed narrative self. But we differ in that I think the narrative is the organising principle of the way objects, memories, and space are interwoven. Individual memories are sometimes retrieved and constructed through interacting with resources in our memory ecology, and the overall narrative helps us to make (historical) sense of the individual memories that build up the narrative, allowing us to see our life as an unfolding trajectoy.

Before moving on to outlining cognitive niche construction theory, two brief points of clarification on the notion of a distributed narrative identity will be helpful. First, one may argue that distributed narrative identities are not necessary properties of selves. It is possible to have a narrative without it being distributed, at least for certain periods of time. A person can dispose of evocative objects, cut ties with transactive memory partners, and spend (parts of) one's life in isolation. Prisoners in solitary confinement, for example, lack access to their memory ecology and during those periods may not have a distributed narrative identity, yet they still have a narrative identity. Such cases are uncommon, to be sure, but nonetheless show that having a distributed narrative identity is not an essential property of selfhood. However, given our highly technological and social lifeworld, most people's memory systems are entangled with evocative objects and transactive memory partners, in that way sustaining their distributed narrative identity. So, distributed narrative identities are contingent properties of selves, depending on our particular sociotechnical situation.

Second, my claim is not that consciousness is extended or distributed. Some philosophers identify self with conscious experience, conceptualising the self as the subject of experience (e.g., Strawson 2017). I'm not claiming that the subject of experience is extended or distributed across agent and environment. My claim is that the building blocks of self-narratives have internal (i.e., neurological and biological) and external (i.e., technological and social) components, suggesting that narratives are distributed. However, the subject of experience, whatever it exactly is, is realised 
by an embodied brain alone. In this sense, my view is perhaps less radical than it may first appear.

\section{Niche construction}

Having outlined how our memory ecology distributes our narrative identity, I now continue with outlining the conceptual framework I will use for better understanding how we interact with and construct our memory ecology.

Niche construction theory is a relatively new approach in evolutionary biology, emphasising the importance of cultural activities for evolutionary processes. "Niche construction refers to the activities, choices, and metabolic processes of organisms, through which they define, choose, modify, and partly create their own niches" (Laland et al. 2000, pp. 132-133). Some organisms have evolved niche construction behaviours, allowing them to alter their local environment which improves their fitness landscapes. Spiders, for example, create webs, beavers build dams, birds make nests, moles dig burrows, and termites build mounds. This kind of tool-use allows animals to actively alter their niche, creating a safer, more regulated environment, which is beneficial for their chances of survival and reproduction, thereby providing an evolutionary benefit.

Once these external structures are built, they can become the source of selection for regulatory, maintenance, and defence behaviours. Some ant and termite species, for instance, regulate the temperature in their mounds through closing the entrances at night to keep the cold air out or change the shape and size of their mound such that it absorbs more sunlight. Such behaviours evolved in response to selection pressures that were initiated by the building of the mounds. Notably, offspring of niche constructing organisms sometimes inherit the environment built by their parents, indicating that niche construction has downstream consequences. In such cases, they don't just inherit genes from their parents but also a modified environment, changing their selection pressures. There are thus two kinds of inheritance: genetic and ecological. Ecological inheritance is important because it allows organisms to modify natural selection pressures, influencing their evolutionary trajectory (Odling-Smee 1988).

\section{Cognitive niche construction}

Humans, too, alter their niches. We are, in fact, niche constructers par excellence, outperforming all other niche constructing species. We build houses, agricultural systems, transportation systems, energy systems, healthcare systems, and many other systems that physically alter our local and non-local environment. A particularly interesting kind of human niche construction is cognitive and epistemic in nature. We don't just alter our physical environment, but also our informational environment (Sterelny 2003, 2004, 2010; Clark 2006; Stotz 2010; Kendall 2011; Sinha 2015; Bertolotti and Magnani 2017). We developed number systems, cartographic systems, diagrams, symbols, alphabets, and languages as well as technologies to 
store that information such as clay tablets, papyrus scrolls, books, newspapers, abacuses, computers, and the internet. An embodied brain embedded in an ecology of informational artifacts is able to achieve and remember vastly more than an embodied brain alone. Such external information-storage systems typically have properties that complement those of the embodied brain (Sutton 2010). External information is usually fixed, discreet, and often more durable, whereas information retained in connectionist networks in the brain is more prone to changing over time (Sutton 2009). Because external information has different properties than information stored in biological memory, we can exploit these differences as to overcome the shortcomings of biological memory, thereby (often) enhancing it.

There is another way in which humans obtain information, namely through social learning. We often learn information and skills from others in our community. Such learned information and skills are important, in part because they can guide niche construction behaviours such as building infrastructure, agriculture, or healthcare systems. There are various social mechanisms or trajectories for transmitting information and skills: (1) vertical (i.e., from parents to offspring), (2) oblique (i.e., from the parental generation but not the parents, e.g., from teachers to students), and (3) horizontal (i.e., within a generation, e.g., from peer to peer) (Laland et al. 2000). Social learning ensures we don't have to invent the same skill, procedure, or technology twice. For example, once the skills, techniques, and tools of agriculture were invented, they spread through social learning, vertically, obliquely, and horizontally.

\section{Downstream epistemic engineering}

Interestingly, as Sterelny (2003) argues, we do not just engineer our current cognitive niche, we also engineer the cognitive niche of the next generation. A distinction can be made between one's personal and public cognitive niche (Sterelny 2010). I engineer my personal cognitive niche, for example with post-it notes, calendars, to-do lists, and notebooks. These cognitive artifacts contain information I created for my own goals. I also often use public informational resources engineered by other people, for example traffic signs, thesauruses, textbooks, and Wikipedia. But in both cases, the informational medium I use to scaffold my memory and cognition is developed by parent generations. Language, numbers, maps, and other symbol systems were developed long ago and passed on to new generations. We were born into the informational environment our parent generation has made, our parent generation was born into the informational environment their parent generation has made, etc. Evolutionary biologists Jablonka and Lamb (2005) refer to the transfer of symbolic information such as language, numbers, and maps from one generation to the next as a "symbolic inheritance system". Importantly, we often improve the informational environment in which we are born, which Sterelny (2003) refers to as "cumulative downstream epistemic engineering". The importance of symbolic inheritance for human culture and cognition can hardly be understated. Merlin Donald, for example, argues that "The memory repositories of culture allow our species to transmit across generations the codes, habits, institutional structures, and 
symbolic memory systems that are needed to operate a significant portion of the processes of modern cognition in human culture" (2000, p. 20).

To give a specific example of cumulative downstream epistemic engineering from distributed cognition theory, consider the classic example of a team of navy sailors trying to navigate a frigate into harbour by using a variety of artifacts such as an alidade, a compass, a log, and a map (Hutchins 1995a). Navigators on each side of the ship take measurements of a distant object with an alidade, which are first written in a $\log$, and then later communicated to the plotter and bearing recorder who jointly triangulate the readings on a map to determine the location of the ship. New sailors learning the navigational process inherit both the practices and cognitive tools from previous generations. Alidades, compasses, logs (including number systems and language), and maps have quite a long developmental history. Alidades were invented in the $16^{\text {th }}$ century, compasses were invented in $200 \mathrm{BC}$ in China, the Hindu-Arabic number system goes back to the $1^{\text {st }}$ century, the Western alphabet was developed in $750 \mathrm{BC}$ in Greece, and the history of cartography goes back at least to $2500 \mathrm{BC}$ and was developed in the ancient Near East. Such navigational instruments and symbol systems have long cultural-evolutionary trajectories. Current navigators therefore stand in a long tradition of instruments, external symbol systems and the cultural practices associated with their use. Navigators don't have to invent the same cognitive technology twice and don't have to figure out how to use the technology because they acquire these skills from their colleagues through (oblique) social learning.

A distinction between cognitive artifact and the representational systems it stores is helpful (Heersmink 2016). A cognitive artifact is a material object or structure that contains and sometimes computes information. Examples include alidades, compasses, rulers, scales, abacuses, notebooks, and digital computers. Representational systems are media for representing information. Charles Saunders Peirce's (1931) tripart distinction between icons, indices, and symbols is useful here to better understand the properties of representational systems. Icons such as maps and scale models are structurally similar to their target domain. A scale model of an airplane is structurally and visually similar to the actual airplane it represents. Indices such as alidades, compasses, and thermometers have a direct causal connection to their target domain. If the target systems changes, the index changes, too. If the temperature increases, the reading on the thermometer changes, too. Symbols such as numbers, words, and sentences primarily obtain their meaning through social conventions, agreement, and shared use. We often improve both the cognitive artifacts and representational systems our parent generation has made. The cultural evolution of representational systems and the artifacts that store and manipulate them has been one of progress and improvement. Languages keep evolving and current computers are much more powerful and efficient than those of the past, roughly doubling their processing powers in 18 months. Likewise, current alidades, compasses, and maps are more efficient and accurate than those used hundreds of years ago.

Socrates expressed concerns about the effects of written language on individual memory and he may well be right, in that writing has transformed our biological memory capacities. Before writing was introduced, information was communicated through oral narratives. The introduction of writing has transformed our ability to remember and articulate long narratives (Ong 1982). Similar claims 
can be made about number systems, maps, and other representational systems. Because these systems allow us to store information externally, we don't have to store it internally, in that way (through lack of practice) weaken some of our memory skills (Sparrow et al. 2011). However, these representational systems allow information to spread horizontally and vertically in a way that spoken language cannot. This has generated an enormous amount of intellectual progress that has been unprecedented in our evolutionary history. Without the horizontal and vertical transfer of information through cognitive artifacts, representational systems, and social learning, human culture and cognition would not be as advanced and sophisticated as they are now. Much progress in the humanities, sciences, law, and government would not be possible without the cultural transfer of information.

Laland and Sterelny (2006) point out that niche construction theorists

seek to explain the adaptive complementarity of organism and environment in terms of a dynamic, reciprocal interaction between the processes of natural selection and niche construction. Evolution is based on networks of causation and feedback in which organisms drive environmental change and organism-modified environments subsequently select organisms (p. 1751).

So, organisms adapt to their environment, but they also adapt the environment, in that way changing some of the selection pressures and giving (human) agency a much more prominent role in evolutionary theory. By externalising memory, we may have influenced the selection pressures on biological memory. The capacity to remember facts and experiences has obvious practical and evolutionary benefits. But there is currently no longer a strong evolutionary selection pressure to store a lot of detailed information in the brain when you can also store that information in the environment. Reliable access to external memory, weakens the selection pressure for a good biological memory. However, because reliable access to external information for the general population significantly increased only with the invention of the printing press (in the 15th century) and became substantial with the invention of the internet and mobile computing devices (in the 20th and 21st century), evolutionary processes may not have had sufficient time to transform the phenotypic trait of biological memory. It is, however, unquestionable that cognition and culture (in general) and biological memory and environmental memory (in particular) interact in various ways (Donald 1991).

A reviewer pointed out that it's possible to be committed to the importance of niche construction as a process without being committed to the importance of ecological inheritance. I support a stronger version of niche construction theory, one that argues that both niche construction as a process and ecological inheritance is important for better understanding how human cognitive evolution occurs. My view is that downstream epistemic engineering must be taken into account if one wants to better understand our cognitive capacities and how those capacities have evolved in relation to the epistemic niche. Ecological inheritance of cognitive artifacts and representational systems is thus central to the development and evolution of our cognitive capacities. 


\section{Narrative niche construction}

Both distributed cognition theory and cognitive niche construction theory focus on changing and using the environment to complete practical cognitive tasks, providing some benefit for the agent. Resources in our memory ecology supporting autobiographical remembering, by contrast, typically aren't used for problem-solving activities. Instead, they are used for thinking about the personal past, which often has identity-supporting functions (Wilson and Ross 2003). There are of course exceptions, but in most cases, we don't use an evocative object or reminisce with a transactive memory partner to solve a practical problem. Autobiographical memory and narrative identity, however, do have practical and evolutionary benefits. Autobiographical narratives provide coherence and structure to our temporal existence. We are organisms that exist over a relatively long period of time, accumulating many experiences. To make sense of these experiences, we organise them in terms of a narrative. Autobiographical narratives help us to make sense of our past, understand the present in terms of an unfolding trajectory, and be future-orientated. These properties of narratives are very important for agents to successfully get around in the world. People with autobiographical memory disorders, including dementia, brain injury, and amnesia often have a diminished agency (Klein et al. 2004). This diminished agency can be partly restored by using evocative objects like SenseCams and lifelogs (Hodges et al. 2011). For these reasons, it's important that we maintain our memory ecology and narrative identity. This section outlines how we interact with and construct our memory ecology by first identifying three types of niche construction processes and then conceptualises how identity-relevant information in objects and (family) stories is transmitted across generations of people.

\section{Cognitive engineering}

Before describing how we construct our memory ecology, it is helpful to briefly characterise the more general notion of cognitive engineering. I will treat cognitive engineering and cognitive niche construction as synonyms, characterising these as altering and utilising the environment for realising our cognitive goals. On this characterisation, cognitive engineering has three main components or stages. The engineering process starts with generating a mental plan for modifying or building an object or structure, typically as a response to a problem situation. Generating a mental plan can be seen as the design stage of the engineering process. This plan is then executed by intentionally acting on the environment as to make an object or structure. The mental plan is typically updated during the making process, allowing for some flexibility and improvisation. Finally, the created object or structure is used for performing a cognitive function, realising the goal(s) of its user(s).

Sometimes these stages are performed by a single person, but more often they are performed by different people or groups of people. When these stages are completed by one person, I refer to it as personal cognitive engineering. An example is making a shopping list. The process starts by generating a mental plan, including an intention, for making the list in response to a problem situation. The problem situation, 
in this case, is an inability to remember a long list of items. The list is then made by an agent, and used to aid remembering buying certain things. When engineering results in publicly available cognitive resources, I refer to it as public cognitive engineering. An example is using traffic signs to navigate. In this case, an agent uses the information on traffic signs to navigate, but designing and creating the traffic signs is performed by others. In cases of public cognitive engineering, an agent only has to generate an intention and then use a pre-existing cognitive artifact. The designing and creating stages are done by others. Cognitive engineering can also be social in nature, in which case an agent creates and uses social structures to achieve his or her cognitive goals. For example, rather than using traffic signs to navigate from A to B, you can ask your friend who is familiar with the area to help you navigate by providing instructions. In this case, you use information stored in someone else's memory to perform a cognitive task.

\section{Creating, editing, using}

Having this general characterisation of cognitive engineering in place, let us move on to narrative engineering, which I characterise as intentionally altering and utilising the environment for aiding us in remembering our personal past. In this section, I identity three types of processes that govern how we interact with our memory ecology, namely creating, editing, and using resources in our memory ecology.

Creating a resource in our memory ecology can be done in a variety of ways. We may intentionally create an evocative object, for example by taking a photo or video, buying a souvenir, or writing an entry in one's journal. We create these objects with the intention to use them later for retrieving and constructing memories. However, associations between an object and a memory are sometimes formed unintentionally. For example, the chair in which I sat when the Netherlands won the European cup may bring back memories of the match, going back to the Piazza Navona may evoke memories of a previous trip to Rome, or a song may evoke memories of your teenage years. In such cases, material culture evokes memories without the object being created for that purpose. These accidental associations between objects and memories are therefore not a form of narrative engineering, because (as outlined in the previous section) engineering requires intentional agency. However, once the association is made, the object can intentionally be used to evoke memories, in which case it is a form of narrative engineering. A musician may, for example, have formed an accidental association between her cello and a show she performed at the Sydney Opera House and because of this association place the instrument in her living room to evoke memories of the show.

With transactive memory systems, it is possible to intentionally create a memory in one's transactive memory partner, for example when asking one's partner to remind one to pay the rent. It is also possible for a dyad to intentionally perform some activity (e.g., museum visit, honeymoon, etc.) with the purpose of creating shared memories. It is, however, more typical for transactive memory systems to work more spontaneously, in that shared memories aren't created intentionally but are the by-product of shared activities. In most cases, one's agency doesn't 
intentionally create memories in a transactive memory partner in the same way as it does in evocative objects.

Editing is intentionally changing the pre-existing informational composition of one's memory ecology. We may do so by removing an evocative object, deleting a photo or video, or putting an evocative object in a box in the garage because we no longer want to be reminded of the memory the object triggers. Lifelogs, too, are frequently edited. Lifelogging technology creates information that is not always useful. SenseCams, for example, create many photos that are irrelevant and need to be deleted as to avoid an information overload. More radical forms of editing are moving to a new house after a loved one passed away as to not be reminded of that person. One may also avoid certain places like restaurants, public squares, etc., as to not be reminded of some person or event. Traumatised people, for example, often avoid the place where the trauma occurred as to not be reminded of the event that caused the trauma. Conversely, one may also undelete photos or videos, put an evocative object from a box in the garage in one's living room, or move to a certain place as to be reminded of past events or life periods. Whilst less common, editing can also occur in transactive memory systems. For example, when reminiscing with one's partner it may become clear that the way your partner understands a shared event is different from how you understand it. Consider the following empirical example from Harris et al. (2014) where a husband and wife reminiscence about a shared experience during a trip they took.

Husband: I remember pulling into that little area and I recall him being a little concerned because it was private property, remember? The big sign that said... Wife: Do no enter!?

Husband: Do no enter. Prosecution may occur.

Wife: And yes. And then he was in his very well marked moose safari couch that would have been very easy to locate again should they wish to have taken action.

Husband: And remember the police car went past and he wouldn't go in until the police car had disappeared.

Wife: Oh is that, oh ok. I didn't quite understand the significance of that, but now...right. Yeah I didn't remember, I didn't understand that at the time.

In this example, the content of a memory of one's transactive memory partner is updated in the light of new information. Whilst this may not necessarily be an intentional process, in that the husband didn't start reminiscing with the goal to edit and update a memory stored in his partner's brain and is therefore not a form of social cognitive engineering, it is a process where the contents of one's memory ecology are changed.

Using is intentionally interacting with an evocative object or transactive memory partner for the purpose of retrieving and constructing an autobiographical memory. Examples of using are browsing through one's photos on one's smartphone, computer, or analogue photo album, visiting a place, playing a song, wearing a piece of jewellery or clothing, or interacting with one's lifelog with the intention to evoke a personal memory. Sometimes autobiographical memories are unintentionally retrieved when interacting with an object or person, for example when watching a 
tv show that reminds you of your recent trip to Italy. Whilst such unintentionally retrieved memories are common, they wouldn't classify as using, because using requires goal-directed intentional agency. Initiating a conversation with one's transactive memory partner with the purpose of reminiscing about the (shared) past can also be seen as a form of using. Quite often, however, conversations with transactive memory partners happen spontaneously. When using resources in our memory ecology, non-occurrent memories are retrieved and reconstructed, thereby reminding us of our personal past and maintaining our narrative identity.

The way we remember our personal past by using resources in our memory ecology has varying levels of specificity. In their seminal paper on memory and self, Conway and Pleydell-Pearce (2000) distinguish between three ways of remembering our personal past, which depend on the degree of specificity. We can, first, remember lifetime periods such as when you went to high school or when you lived in Sydney. Second, we can remember general events, which may be repeated events such as going to your favourite restaurant on Fridays or single events like your recent trip to Italy for a conference. Third, we can remember specific experiences like your first day at school, moving to a new city, or giving a presentation at a conference. Autobiographical memories thus have different levels of abstraction. Moreover, memories of past experiences can be both semantic and experiential. I may, for example, merely remember that I did a subject in molecular biology twenty years ago but don't have any specific experiential memories of the lectures and exams. However, memories can also be imbued with meaning, affect, and have a rich phenomenology, for example the memory of your $\mathrm{PhD}$ graduation in which you remember parts of the dean's speech, how you felt, what the room looked like, who was there with you, what you did afterwards, etc. Our personal past can thus be remembered both semantically and experientially. Interacting with resources in one's memory ecology can trigger semantic and experiential memories, and might be of short or long lifetime periods, of general or specific events, or of specific experiences.

In their empirical work on evocative objects, Kirk and Sellen (2010) examined what types of objects trigger autobiographical memories. They conducted an ethnography of evocative objects in which they went to people's homes and observed and interviewed the inhabitants who had to select a number of evocative objects from each room and explain why and how they were autobiographically meaningful. They distinguish between three types of object storage, which they refer to as display, functional storage, and deep storage. When objects are on display, they are located in easily visible places, usually in the living room. Due to their location, people will perceive the object frequently, thus also frequently triggering memories. The main function of objects on display is not practical but to trigger memories. When objects are in functional storage, they are typically located in less prominent places and will trigger memories only when the object is used. An example Kirk and Sellen mention is an inherited ladle located in a kitchen drawer. The participant points out that only when the ladle is used it occasionally triggers memories of her deceased grandmother. The main function of objects in functional storage is often not to trigger memories but is more practical. The evocative powers of such objects are secondary to their practical functions. Objects in deep storage are rarely interacted with and often put in boxes in the attic or garage. There are various reasons 
for putting objects in deep storage, for example for aesthetics reasons, protecting the object, reducing clutter, or because it's associated with a painful or private memory. The main function of objects in deep storage is often to trigger memories, they are not used for practical functions. This trichotomy of types of object storage shows that evocative objects are used in different ways and for different reasons.

This section has demonstrated that our memory ecologies are dynamic, reflecting our changing narrative identity. Our narrative identity changes over time in at least two ways. First, we have new experiences that we weave into our existing narrative. Memories of these new experiences are supported by adding new evocative objects (e.g., photos, videos, souvenirs, books, etc.) to our memory ecology and sometimes putting these on display. Second, the way we look at events in our personal past changes over time, in that some events become less self-defining over time. For example, after going on a long-planned safari to Africa, memories of the trip may become an important part of one's narrative identity. Due to the importance of the event, someone may put pictures or mementos of the trip on display in one's living room. But after time passes and the event is deeper in one's past, one may come to see the trip as less self-defining and then put the photos and mementos in deep storage. Our changing narrative identity is thus supported by a dynamic memory ecology.

\section{Downstream narrative niche construction}

The examples discussed in the previous section are mainly concerned with narrative niche construction in the here-and-now, i.e., with horizontal information trajectories between agents and objects or agents and agents. But narrative niche construction also happens on longer timescales and can have downstream effects. Downstream narrative niche construction occurs when evocative objects and (family) stories are passed on from one generation to the next. We often inherit meaningful objects from our parents who sometimes have inherited those objects from their parents. One example Petrelli and Whittaker (2010) mention is a box (kept in deep storage) given to a subject by her mother filled with personal objects. The subject says:

This was given to me by my mother, last Christmas. She picked up all sorts of lovely little family treasures: pictures of my great grandparents, my great grandmother's sewing things, my great uncles wooden carvings and all sorts of old family things. It's like a little corner of part of my life (2010, p. 161).

Likewise, we also "inherit" stories from our parents, grandparents, and other family members (McAdams 2004). Social psychologists Michael Pratt and Barbara Fiese point out that "Family stories are one way in which individuals connect across generations and create a sense of family history and identity" (2004, pp. 2-3). Sometimes these stories are about our own past. We often don't remember our experiences before age 4 and have very few memories of the period between age 4 and 10 , which is referred to as childhood amnesia. We learn about this period from our parents who narrate our past events to us. Consider the following dialogue between John who is an 8-year-old boy and his mother from Fivush et al. (2011). 
John: Um I was born in the Holy Cross Hospital in Weinheim hmmm, what happened then Mum?

Mother: (Laughs) Yeah then you were born [John: Yeah] Then we went home [John: Yeah and then] That was the first two years, you don't know [John: Yeah] Then we were we were still living at Gran's the first three months [John: Yeah] And as our flat wasn't ready yet you can't remember that [John: Yeah]. Then we moved with you to the flat in B [John: Hmm] Dad, you, and me [John: Hmm] And then we lived there for a while [John Yeah]

John: And then you split up

Mother: Right

John: And then Mum? What happened then?

Mother: Perhaps we should tell a little bit about what it was like while you we were living together that you can't really remember

John: Yeah what was it like then?

Mother: I got my training, I got it quite early, we were pretty young.

In this example, John's mother narrates events from his past to him such that he can incorporate those into his narrative. Such mother-child dyads can be seen as an intergenerational transactive memory system, in which the mother functions as the autobiographical memory system for the child, thereby scaffolding the content of the child's narrative. Importantly, parents do not just scaffold the content of the narrative, but also scaffold the narrative skills of their children. As soon as children can talk, parents encourage them to narrate their past by asking and elaborating on their past activities (Reese 2002). Parents continue to scaffold the development of their children's narrative capacities throughout childhood and adolescence. In parent-child dyads, there are thus social mechanisms for the vertical transmission of both narrative content and narrative skills, which can be seen as a form of downstream narrative niche construction. More generally, family stories can provide material for the formulation of personal and narrative identity. Grandchildren may, for example, with pride and a sense of family connection, have incorporated stories of their grandparents' ambitious and successful migration to their new home country (Pratt and Fiese 2004).

Kirk and Sellen (2010) studied how objects are passed on from one generation to the next. One participant inherited a book of jam recipes from her deceased grandmother, reminding her of her shared experiences with her grandmother. Another participant keeps inherited photos of deceased relatives so that her children know about these relatives, in that way fostering a connection between family members and a shared family history. The participant says: "I'm quite aware of that whole thing of, erm, them being aware of their identity and their history and where they've come from, I think that's really important for children to know that" (Kirk and Sellen 2010 , p. 21). In these examples, identity-relevant information is passed on from one generation to the next, in that way generating vertical information trajectories which constitute a part of our memory ecologies, scaffolding our narrative identity.

Downstream narrative niche construction is not necessarily cumulative. Improvements made to cognitive technologies and representational systems (e.g., more accurate compasses, new words, new scientific symbols, new programming languages, 
updated maps, new ways of representing knowledge, etc.) often first spread horizontally and then also vertically and obliquely throughout a population. Because this kind of niche construction is cumulative, the new generation keeps making improvements to the inherited cognitive technologies and representational systems. For example, computer systems keep improving and natural languages keep evolving. By contrast, inherited objects and (family) stories are possibly passed on a few generations and so the cultural lineage or trajectory of those objects and stories is typically much shorter as compared to traditional lineages of cognitive technologies and representational systems. Religious texts such as the Vedas, bibles, and Korans, for example, have been passed on for hundreds of generations. Downstream narrative niche construction is thus often not cumulative, in the sense that we usually don't make improvements to the content and functions of inherited objects and (family) stories, although the meaning of objects and content of stories may, of course, change when passed on to the next generation.

\section{Conclusion}

This paper has conceptualised how we engineer our memory ecology and manage our distributed narrative identities by using a cognitive niche construction approach. I first argued that our autobiographical memory systems are distributed, in that we often remember our personal past by interacting with evocative objects and transactive memory partners. I then argued our identity is constituted by our narrative and that this narrative is constructed by interacting with evocative objects and other people. Our narrative identity, or so I claimed, is relational and distributed across our brains, bodies, and environmental resources. I ended with identifying three types of niche construction processes that govern how we interact with our memory ecology, namely creating, editing, and using resources in our memory ecology. I also conceptualised how identity-relevant information in objects and (family) stories is transmitted vertically, that is, across generations of people. Identifying these processes allows us to better understand the cultural information trajectories that constitute our memory ecologies. Thus, what I argued in this paper is that our memory ecology distributes our narrative identity and that engineering our memory ecology is a form of narrative niche construction.

Acknowledgements I like to thank the two anonymous reviewers for their helpful comments.

\section{References}

Adams F, Aizawa K (2008) The bounds of cognition. Wiley-Blackwell, Oxford

Bell G, Gemmell J (2009) Total recall: how the e-memory revolution will change everything. Dutton, New York

Bertolotti T, Magnani L (2017) Theoretical considerations on cognitive niche construction. Synthese 194(12):4757-4779

Bietti L, Sutton J (2015) Interacting to remember at multiple timescales: coordination, collaboration, cooperation and culture in joint remembering. Interact Stud 16(3):419-450 
Clark A (2003) Natural born cyborgs: minds, technologies, and the future of human intelligence. Oxford University Press, Oxford

Clark A (2006) Language, embodiment, and the cognitive niche. Trends Cogn Sci 10(8):370-374

Clark A (2008) Supersizing the mind: embodiment, action and cognitive extension. Oxford University Press, Oxford

Clark A, Chalmers D (1998) The extended mind. Analysis 58(1):10-23

Conway M, Pleydell-Pearce C (2000) The construction of autobiographical memories in the selfmemory system. Psychol Rev 107(2):261-288

Dipert R (1993) Artifacts, art works, and agency. Temple University Press, Philadelphia

Donald M (1991) Origins of the modern mind: three stages in the evolution of culture and cognition. Harvard University Press, Cambridge

Donald M (2000) The central role of culture in cognitive evolution: a reflection on the myth of the isolated mind. In: Nucci L, Saxe G, Turiel E (eds) Culture, thought and development. Erlbaum, Mahwah, pp 39-48

Fasoli M (2018) Substitutive, complementary and constitutive cognitive artifacts: developing an interaction-centered approach. Rev Philos Psychol 9:671-687

Fivush R, Habermas T, Waters T, Zaman W (2011) The making of autobiographical memory: intersections of culture, narratives and identity. Int J Psychol 46(5):321-345

Gallagher S (2000) Philosophical conceptions of the self: implications for cognitive science. Trends Cogn Sci 4(1):14-21

Habermas T, Ehlert-Lerche S, de Silveira C (2009) The development of the temporal macrostructure of life narratives across adolescence: beginnings, linear narrative form, and endings. J Pers 77(2):527-560

Harris C, Barnier A, Sutton J, Keil P (2014) Couples as socially distributed cognitive systems: remembering in everyday social and material contexts. Mem Stud 7(3):285-297

Heersmink R (2013) A taxonomy of cognitive artifacts: function, information, and categories. Rev Philos Psychol 4(3):465-481

Heersmink R (2016) The metaphysics of cognitive artefacts. Philos Explor 19(1):78-93

Heersmink R (2017) Distributed selves: personal identity and extended memory systems. Synthese 194(8):3135-3151

Heersmink R (2018) The narrative self, distributed memory, and evocative objects. Philos Stud 175(8):1829-1849

Heersmink R (2020) Varieties of the extended self. Conscious Cogn 85:103001

Heras-Escribano M (2020) The evolutionary role of affordances: ecological psychology, niche construction, and natural selection. Biol Philos 35:30

Hodges S, Berry E, Wood K (2011) SenseCam: a wearable camera that stimulates and rehabilitates autobiographical memory. Memory 19(7):685-695

Houkes W, Vermaas P (2010) Technical functions: on the use and design of artefacts. Springer, Dordrecht

Hutchins E (1995a) Cognition in the wild. MIT Press, Cambridge

Hutchins E (1995b) How a cockpit remembers its speed. Cogn Sci 19(3):265-288

Hutchins E (1999) Cognitive artifacts. In: Wilson R, Keil F (eds) The MIT encyclopedia of the cognitive sciences. MIT Press, Cambridge, pp 126-128

Hutchins E (2010) Cognitive ecology. Top Cogn Sci 2(4):705-715

Jablonka E, Lamb M (2005) Evolution in four dimensions: genetic, epigenetic, behavioral and symbolic variation in the history of life. MIT Press, Cambridge

Jongepier F (2016) Towards a constitutive account of implicit narrativity. Phenomenol Cogn Sci 15(1):51-66

Kendall J (2011) Cultural niche construction and human learning environments: investigating sociocultural perspectives. Biol Theory 6(3):241-250

Kirk D, Sellen A (2010) On human remains: values and practice in the home archiving of cherished objects. ACM Trans Comput Hum Interact 17(3):1-43

Kirsh D, Maglio P (1994) On distinguishing epistemic from pragmatic action. Cogn Sci 18:513-549

Klein S, German T, Cosmides L, Gabriel R (2004) A theory of autobiographical memory: necessary components and disorders resulting from their loss. Soc Cogn 22:460-490

Kroes P (2012) Technical artefacts: creations of mind and matter-a philosophy of engineering design. Springer, Dordrecht 
Krueger J (2011) The who and the how of experience. In: Zahavi D, Thompson E, Siderits M (eds) Self, no-self? Perspectives from analytical, phenomenological, and Indian traditions. Oxford University Press, Oxford, pp 27-55

Laland K, Sterelny K (2006) Perspective: seven reasons (not) to neglect niche construction. Evolution 60(9):1751-1763

Laland K, Odling-Smee J, Feldman M (2000) Niche construction, biological evolution, and cultural change. Behav Brain Sci 23:131-175

Loftus E (2003) Make-believe memories. Am Psychol 58(11):867-873

Lupton D (2016) The quantified self: A sociology of self-tracking. Polity Press, Cambridge

Mackenzie C, Poltera J (2010) Narrative integration, fragmented selves, and autonomy. Hypathia 25(1):31-54

Margolis E, Laurence S (eds) (2007) Creations of the mind: theories of artifacts and their representation. Oxford University Press, Oxford

McAdams D (2004) Generativity and the narrative ecology of family life. In: Fiese B, Pratt M (eds) Family stories and the life course across time and generations. Erlbaum, Mahwah, pp 235-257

Meijers A (ed) (2009) Philosophy of technology and engineering sciences. Elsevier, Amsterdam

Menary R (2008) Embodied narratives. J Conscious Stud 15(6):63-84

Menary R (2010a) Introduction: the extended mind in focus. In: Menary R (ed) The extended mind. MIT Press, Cambridge, pp 1-25

Menary R (ed) (2010b) The extended mind. MIT Press, Cambridge

Michaelian K, Sutton J (2013) Distributed cognition and memory research: history and current directions. Rev Philos Psychol 4(1):1-24

Mitchell W (2007) The Melbourne train. In: Turkle S (ed) Evocative objects. MIT Press, Cambridge, pp $145-152$

Newen A, De Bruin L, Gallagher S (eds) (2018) The Oxford handbook of 4E cognition. Oxford University Press, Oxford

Norman D (1993) Things that make us smart: defending human attributes in the age of the machine. Addison-Wesley Publishing, Reading

Odling-Smee J (1988) Niche constructing phenotypes. In: Plotkin H (ed) The role of behaviour in evolution. MIT Press, Cambridge, pp 31-79

Odling-Smee J, Laland K, Feldman M (2003) Niche construction: the neglected process in evolution. Princeton University Press, Princeton

Ong W (1982) Orality and literacy: the technologizing of the word. Methuen, London

Pascuzzi D, Smorti A (2017) Emotion regulation, autobiographical memories and life narratives. New Ideas Psychol 45:28-37

Peirce C (1931) The collected papers, vol 1-6. Harvard University Press, Cambridge

Petrelli D, Whittaker S (2010) Family memories in the home: contrasting physical and digital mementos. Pers Ubiquit Comput 14(2):153-169

Petrelli D, Whittaker S, Brockmeier J (2008) Autotopography: What can physical mementos tell us about digital memories? In: CHI '08 ACM conference on Human factors in computing systems, pp 53-62

Piredda G (2020) What is an affective artifact? A further development of situated affectivity. Phenomenol Cogn Sci 19(3):549-567

Pratt M, Fiese B (2004) Families, stories, and the life course: an ecological context. In: Fiese B, Pratt M (eds) Family stories and the life course across time and generations. Erlbaum, Mahwah, pp 1-24

Preston B (2018) Artifact. In: Zalta E (ed) Stanford encyclopedia of philosophy. URL: https://plato.stanf ord.edu/entries/artifact/

Reese E (2002) A model of the origins of autobiographical memory. In: Fagen J, Hayne H (eds) Progress in infancy research. Lawrence Erlbaum Associates, Mahwah, pp 215-260

Ricoeur P (1984) Time and narrative, vol 1. University of Chicago Press, Chicago

Robbins P, Aydede M (eds) (2008) The Cambridge handbook of situated cognition. Cambridge University Press, Cambridge

Rowlands M (2010) The new science of the mind: from extended mind to embodied phenomenology. MIT Press, Cambridge

Rudd A (2009) In defence of narrative. Eur J Philos 17(1):60-75

Rupert R (2004) Challenges to the hypothesis of extended cognition. J Philos 101(8):389-428

Rupert R (2010) Cognitive systems and the extended mind. Oxford University Press, Oxford

Schacter D (2001) The seven sins of memory: how the mind forgets and remembers. Houghton Mifflin, Boston 
Schechtman M (1996) The constitution of selves. Cornell University Press, Ithaca

Schechtman M (2005) Personal identity and the past. Philos Psychiatry Psychol 12(1):9-22

Schechtman M (2014) Staying alive: personal identity, practical concerns, and the unity of a life. Oxford University Press, Oxford

Sinha C (2015) Language and other artifacts: socio-cultural dynamics of niche construction. Front Psychol 6:1601. https://doi.org/10.3389/fpsyg.2015.01601

Sparrow B, Liu J, Wegner D (2011) Google effects on memory: cognitive consequences of having information at our fingertips. Science 333(6043):776-778

Steffensen S (2017) Human interactivity: problem-finding, problem-solving, and verbal patterns in the wild. In: Cowley S, Vallée-Tourangeau F (eds) Cognition beyond the brain. Springer, Dordrecht, pp $85-103$

Sterelny K (2003) Thought in a hostile world: the evolution of human cognition. Blackwell, Oxford

Sterelny K (2004) Externalism, epistemic artefacts and the extended mind. In: Schantz R (ed) The externalist challenge. De Gruyter, Berlin, pp 239-254

Sterelny K (2010) Minds: extended or scaffolded? Phenomenol Cogn Sci 9(4):465-481

Sterelny K (2014) Constructing the cooperative niche. In: Barker G, Desjardins E, Pearce T (eds) Entangled life: history, philosophy and theory of the life sciences. Springer, Dordrecht, pp 261-279

Stotz K (2010) Human nature and cognitive-developmental niche construction. Phenomenol Cogn Sci 9(4):483-501

Strawson G (2004) Against narrativity. Ratio 17(4):428-452

Strawson G (2017) The subject of experience. Oxford University Press, Oxford

Sutton J (2009) Remembering. In: Robbins P, Aydede M (eds) The Cambridge handbook of situated cognition. Cambridge University Press, Cambridge, pp 217-235

Sutton J (2010) Exograms and interdisciplinarity: history, the extended mind, and the civilizing process. In: Menary R (ed) The extended mind. MIT Press, Cambridge, pp 189-225

Tribble E, Sutton J (2011) Cognitive ecology as a framework for Shakespearean studies. Shakespear Stud 39:94-103

Turkle S (ed) (2007) Evocative objects: things we think with. MIT Press, Cambridge

Wegner D (1986) Transactive memory: a contemporary analysis of the group mind. In: Mullen B, Goethals G (eds) Theor Group Behav. Springer, New York, pp 185-208

Wilson A, Ross M (2003) The identity function of autobiographical memory: time is on our side. Memory $11(2): 137-149$

Zahavi D (2010) Minimal self and narrative self: a distinction in need of refinement. In: Fuchs T, Sattel H, Henningsen P (eds) The embodied self: dimensions, coherence, and disorders. Schattauer, Stuttgart, pp 3-20

Publisher's Note Springer Nature remains neutral with regard to jurisdictional claims in published maps and institutional affiliations. 